\title{
Protective effect of apricot (Prunus armeniaca $L$.) and its kernel against paracetamol-induced hepato-renal toxicity in rats
}

\author{
Zakia M. Abdelkader*, Lamiaa A.A Barakat** and Mai E.Abdelkawi* \\ *Biochemistry and Nutrition Department- Women's College, Ain Shams University \\ ** Chemistry Department- Science College, Port Said University
}

\begin{abstract}
This study was designed to investigate the protective effect of fresh, dried apricot and its kernel added to diet (10\% and 20\% w/w) on paracetamol ( $\mathrm{N}$-acetyl-p-aminophenol , APAP)-induced cellular toxicity in rats. Eighty male albino rats were used in this study, divided into 8 groups 10 rats each. Gp (1): set as healthy control, Gp (2) set as APAPaffected control. Gps (3-4) fed on diet supplemented with fresh apricot $(10 \%$ \& $20 \%$ wlw), Gps (5-6) fed on diet supplemented with dried apricot (10\% \& 20\% wiw). Gps (78 ) fed on diet supplemented with apricot kernel (10\% \& 20\% wlw). After 6 weeks, rats from groups (2:8) were injected with a single dose, i.p injection, of APAP (500 mglkg body weight). After $24 \mathrm{~h}$ of injection, rats were sacrificed under diethyl ether anesthesia. Serum and tissue samples were collected to perform the biochemical analysis. Serum liver function tests [total protein, albumin, total bilirubin, direct and indirect bilirubin, the activities of alanine amino transferase (ALT), asparatet amino transferase (AST) and alkaline phosphatase (ALP)] were measured. Moreover, serum kidney function tests (urea, creatinine, and uric acid), and serum lipids profile were measured. Also, antioxidant status was evaluated by measuring reduced glutathione (GSH) and malondialdehyde (MDA) in liver and kidney homogenate. The results were supported with microscopic examination of liver and kidney tissues. The results of this study revealed that administration of APAP caused marked reduction in serum total protein, albumin and tissue GSH, in association with elevation of serum bilirubin, activities of AST, ALT, ALP, urea, creatinine, uric acid, lipids profile, and tissue MDA. On the other hand, supplementation of diet with fresh, dried apricots and apricot kernel had protective effect on liver and kidney tissues that was manifested by improving liver and kidney functions, antioxidant status, and lipids profile. The microscopic examination of liver and kidney tissues showed also amelioration in the appearance of cells. The protective effect of apricot and its kernel is due to their antioxidant potential that is represented by their rich content of dietary fibers, $\beta$-carotene, vitamin $\mathrm{C}$, tocopherols, flavonoids, poly unsaturated fatty acids, and some polyphenols that appears to protect against reactive oxygen species (ROS) and consequently APAP toxicity.
\end{abstract}

Key Words: Paracetamol, apricot, apricot kernel, liver, kidney, antioxidants, oxidative stress, lipids profile.

Corresponding author:Mai_omara2002@yahoo.com 


\section{Introduction:}

Paracetamol (Acetaminophen, N-acetyl-p-aminophenol, APAP) was introduced worldwide in the 1950s as an antipyretic and analgesic drug. Paracetamol is a widely used over-the-counter analgesic (pain reliever) and antipyretic (fever reducer) drug. Paracetamol is primarily metabolized by the liver, most of which are inactive, non-toxic and eventually excreted by the kidneys. But, excessive use of paracetamol can damage multiple organs, and the toxicity is not from the drug itself but from one of its metabolites, N-acetyl-p-benzoquinoneimine (NAPQI) (Liu et al., 2011).

Therapeutic doses of APAP of approximately $4 \mathrm{~g}$ daily are considered to be safe while overdose produces severe liver injury that can lead to fatal fulminant hepatic failure $(\boldsymbol{G u l}$ et al., 2012). At therapeutic doses, 60-90\% of the APAP is metabolized into nontoxic glucuronide and sulfate by conjugation reaction, $5-10 \%$ is oxidized into N-acetyl-pbenzoquinoneimine (NAPQI) by mixed-function oxidase enzymes, by cytochrome P-450, which is immediately conjugated with glutathione (GSH) and subsequently excretes as cysteine and mercapturate conjugates. At exposure to high dose APAP, much more NAPQI is formed; subsequently the hepatic GSH stores are consumed. Eventually, the remaining NAPQI binds to cellular macromolecules covalently and leads to cell death. Only $1-4 \%$ of a therapeutic dose of APAP is excreted unchanged in urine. The NAPQI is believed to have two separate but complementary effects by which hepatocellular injury is induced; firstly, it reacts with GSH thereby depleting normal defense of hepatic cells against oxidative damage, secondly, it is a potent oxidizing and arylating agent that inactivates key sulphydryl groups of certain enzymes (particularly those controlling calcium homeostasis). The APAP-induced renal damage probably results from the formation of NAPQI, though in the kidney, NAPQI is generated by prostaglandin endoperoxide synthetase rather than by CYP-dependent mixed-function oxidases. The binding of NAPQI with cellular proteins leads to necrosis (Bhadauria, 2010; and Abirami et al., 2015).

Therefore, in recent years, searching of protective molecules that would offer maximum protection against APAP induced hepatic and renal damages is required. Although several antioxidant molecules (such as $\mathrm{N}$-acetyl-cysteine, taurine, melatonin, vitamins $\mathrm{E}$ and $\mathrm{C}$, etc.) have been used to prevent APAP-induced hepatotoxicity, offer protection against APAP-induced nephrotoxicity. Consumption of fruits as a significant portion of our daily diets has been associated with lower risks of different diseases. Further, fruits are high in health promoting phytonutrients, antioxidants, flavonoids (anthocyanins, flavonols and proanthocyanidins), and polyphenols (Das et al., 2010).

Apricot (Prunus armeniaca L.) is one of the most important commercial crops and worldwide preferable in the markets of production and consumption. Apricot is mostly suitable for manufacturing in many commercial forms such as fresh fruit, dried fruit and fruit juice. Despite the wide cultivation in many parts of the world, there is very little knowledge about the potential health benefits of apricot consumption. Since, apricot includes flavonoids and carotenoids, it may have antioxidant properties. Beta carotene is the precursor of vitamin A that appears to protect against reactive oxygen species (ROS). 
Also, apricot kernel is an important source of antioxidants (Yurt \& Celik, 2011). The apricot fruits are a rich source of oil, protein, soluble sugars, fibers as well as fatty acid, carotenoids such as $\beta$-carotene, $\beta$-cryptoxanthin, $\gamma$-carotene, and lycopene, phenolics such as chlorogenic and neochlorogenic acids, (+)-catechin and (-)-epicatechin, and rutin, pectin and mineral elements including $\mathrm{Na}, \mathrm{P}, \mathrm{K}, \mathrm{Ca}, \mathrm{Mg}, \mathrm{Fe}, \mathrm{Zn}, \mathrm{Mn}$, and $\mathrm{Cu}$ (Orhan \& Kartal, 2011). Moreover, apricot kernel is an important source of dietary protein as well as oil and fiber. The kernel is added to bakery products as whole kernel or grounded and also consumed as appetizers. Apricot kernels are used in the production of oils, benzaldehyde, cosmetics, active carbon, and aroma perfume (Durmaz \& Alpaslan, 2007). It is well known that apricot kernels contain a wide variety of bioactive components, and consumption of apricot kernel has been associated with a reduced risk of chronic diseases. Interestingly, apricot kernels contain as much as 50\% oil. Apricot kernel oil (AO) is rich in mono- and polyunsaturated fatty acids, with oleic and linoleic acids being the major constituents, and a number of minor components, such as tocopherols and phenolic compounds. Monounsaturated and polyunsaturated fatty acids, as well as minor lipid components, play an important role in human nutrition and health. Diets rich in these compounds can decrease blood pressure and total blood cholesterol levels, fight oxidative stress and maintain body weight (Zhang et al., 2011).

This study was designed to investigate the protective effect of fresh, dried apricots and apricot kernel against paracetamol induced hepato-renal toxicity.

\section{Materials and Methods:}

\section{1- Materials:}

Male adult albino rats (Spargue Dawly strain) weighing115 $\pm \mathrm{g}$ were obtained from Breeding Unit of the Egyptian Organization for Biological Products and Vaccines (Helwan, Egypt). Standard balanced diet was used and prepared according to AIN 1993 and adjusted by Reeves et al., (1993). The fresh mature apricot fruit, (Prunus armeniaca L.) from Rosaceae strain, was purchased from El- Oubour market (Cairo, Egypt), since season May to August 2012. Also, sulphited dried apricot was purchased from local markets. Moreover, apricot kernel was taken off from fresh apricot and ground. Fresh, dried apricot and kernel were ground separately by using food processor grinder and added to standard balanced diet as 10 or $20 \% \mathrm{w} / \mathrm{w}$. Paracetamol (APAP) was purchased from Alexandria Company, for Pharmaceuticals \& Chemical industries by dose of 500 $\mathrm{mg} / \mathrm{kg}$ body weight according to Kantham, (2011).

\section{2- Methods:}

\subsection{1-Animal trial:}

Eighty adult male albino rats Spargue Dawly strain weighing $115 \pm 5 \mathrm{~g}$ were randomly housed individually with constant controlled environments in stainless steel cages; temperature $25^{\circ} \mathrm{C} \pm 3^{\circ} \mathrm{C}$ and $12 / 12 \mathrm{~h}$ light/dark cycle were held. Experimental animals were fed with standard balanced diet and water ad libitum for a one week acclimitation period, then the eighty rats were divided into eight groups (10 per each) as follow: Group (1) set as normal healthy control rats (-ve control), Group (2) set as 
APAP-control (+ve control). Groups (3-4) fed on diet supplemented with fresh apricot $(10 \% \& 20 \%$ wlw), Groups (5-6) fed on diet supplemented with dried apricot $(10 \%$ \& $20 \%$ wlw). Groups (7-8) fed on diet supplemented with apricot kernel $(10 \% \& 20 \%$ wlw).

Diet was introduced to the rats in special cups, water was introduced in a well-sealed bottles. All rats had free access to food and water all times for six weeks. Food intake was recorded daily. The animals were weighed weekly to monitor the body weight changes. At the end of 6 weeks, -ve control rats were i.p. injected with a single dose of saline, while all other groups of rats were i.p. injected with single dose of freshly prepared APAP in saline at a dose $500 \mathrm{mg} / \mathrm{kg}$ body weight according to Kantham, (2011). After $24 \mathrm{~h}$ of APAP injection and $12 \mathrm{~h}$ fasting, the animals were scarified under ether anesthesia and blood samples were collected directly from portal vein. The blood samples were left to clot and the serum was separated by cooling centrifugation $\left(4^{\circ} \mathrm{C}\right)$ at $3000 \mathrm{rpm}$ for 10 minutes and kept at $-20^{\circ} \mathrm{C}$ until analysis. The organs (liver and kidney) were separated immediately, rinsed and washed in saline solution then blotted on filter paper to remove water residue then weighed, part of liver and kidney specimens were preserved in $10 \%$ formalin for histopathological examination. The other part of liver and kidney from each group were stored at $-20^{\circ} \mathrm{C}$ until analysis.

\subsection{2- Biochemical analysis:}

Total proteins in serum were determined according Gornal, et al., (1949). Serum albumin was measured by colorimetric method of Doumas et al., (1971). Serum total, direct, indirect bilirubin was measured by colorimetric method of Walter \& Gerade, (1970). AST and ALT activities were measured by colorimetric method of Reitman \& Frankel, (1957). ALP activity was measured by colorimetric method of Belfield \& Goldberg, (1971). Urea and Creatinine were determined in serum by urease-Berthelot method of Fawcett \& Soctt, (1960) and Bartels et al., (1972) respectively. Uric acid was measured by enzymatic colorimetric method according to Barham \& Trinder, (1972). Serum total cholesterol (TC) was determined enzymatically according to Allain et al., (1974). Triacylglycerols (TAG) was measured enzymatically according to Fossati \& Principe, (1982). High density lipoprotein (HDL-C), low density lipoprotein (LDL-C) and very low density lipoprotein (VLDL-C) were determined according to Friedewald, (1972). Liver and kidney MDA was determined according to Draper \& Hadley (1990), by a thiobarbituric acid method. The determination of liver and kidney GSH was measured according to Beutler et al. (1963).

\subsection{Histopathological Examination:}

Liver and kidney specimens from each group were embedded in paraffin wax and the microscopic sections of $5 \mu \mathrm{m}$ were taken and stained with hematosylin and eosin $(\mathrm{H} \& \mathrm{E}$ stain) for histological examination (Drury \& Wallington, 1980).

\subsection{Statistical Analysis:}


The data were statistically analyzed according to Steel \& Torrie (1980) using SPSS computer Program. The results were presented as mean \pm SE. The differences between mean values were determined by analysis of variance (ANOVA test), followed by Duncan's multiple rank test using MSTAT-C computer program. Statistical significance of the relationships between variables was calculated by linear regression analysis, where $\mathrm{P} \leq 0.05$ was considered significant.

\section{Results and Discussion}

\subsection{1- Biochemical Results:}

Data in table (1) of nutritional evaluation showed that, there was no significant increase in food intake and body weight gain in APAP-control rats compared to healthy rats. Also, it was noticed from results that supplementation with fresh and dried apricot $(10 \& 20 \%)$ before APAP administration caused significant increase $(\mathrm{p} \leq 0.05)$ in food intake and body weight gain compared to APAP- control rats. On the other hand, supplementation with apricot kernel $(10 \& 20 \%)$ caused significant decrease $(p \leq 0.05)$ in food intake and body weight gain as compared to APAP-control rats. In addition, there was no significant change in relative organs weight between all groups.

It is clear form table (2) of liver function measurements, there were significant decrease $(\mathrm{p}<0.05)$ in serum total protein and albumin levels in APAP-control rats as compared with healthy rats. Furthermore, there were significant increase $(\mathrm{p}<0.05)$ in serum total protein and albumin levels in groups pre-treated with fresh apricot, dried apricot and apricot kernel as compared to APAP-control group. Our results provided that the protective effect of fresh and dried apricot was dose dependent. The data represented in table (2) also showed that there was significant increase $(\mathrm{p}<0.05)$ in serum total, direct, and indirect bilirubin as compared to healthy control rats. On the other hand, supplementation of diet with fresh apricot, dried apricot, and apricot kernel before APAP injection caused significant decrease $(\mathrm{p}<0.05)$ in serum total, direct, and indirect bilirubin as compared to APAP-control rats. Generally, the improvement in serum bilirubin level was dose dependent.

The statistical analysis in table (3) of serum liver enzymes indicated that there was a significant increase $(\mathrm{p}<0.05)$ in serum AST, ALT, and ALP activities in APAP-control rats as compared to healthy control rats. It is also clear that there were significant decrease $(\mathrm{p}<0.05)$ in serum AST, ALT, and ALP activities in rats fed on fresh apricot, dried apricot, and apricot kernel supplemented diet as compared to APAP-control rats. These data proved that the protective effect of fresh and dried apricot was dose dependent.

Statistical analysis in table (4) of kidney function measurements showed that administration of APAP caused significant increase $(p<0.05)$ in serum urea, creatinine, and uric acid levels as compared to healthy control rats. On the other hand, the statistical analysis illustrated that rats fed on fresh apricot, dried apricot and apricot kernel supplemented diets showed significant decrease $(\mathrm{p}<0.05)$ in serum urea, creatinine, and 
uric acid levels as compared to APAP-control rats. The data proved that the protective effect of fresh and dried apricot was dose dependent.

Results from table (5) of serum lipids profile concluded that APAP caused marked elevation $(\mathrm{p}<0.05)$ in serum TC, TAG, LDL-C, and VLDL-C levels and significant reduction $(\mathrm{p}<0.05)$ in serum HDL-C level as compared to healthy rats. The statistical analysis also indicated that supplementation of rat's diets with fresh apricot, dried apricot, and apricot kernel by levels $(10 \& 20 \%)$ caused significant decrease $(\mathrm{p}<0.05)$ in serum TC, TAG, LDL-C, and VLDL-C levels and significant increase $(\mathrm{p}<0.05)$ in serum HDL$\mathrm{C}$ level as compared to APAP-control rats. the hypolipidemic effect of fresh, dried apricot and apricot kernel was dose dependent.

The results in table (6) showed disturbance of antioxidant status in APAP administered rats which indicated by significant decrease $(\mathrm{p}<0.05)$ in liver and kidney GSH and significant increase $(\mathrm{p}<0.05)$ in liver and kidney MDA levels in a comparison with healthy rats. Moreover, supplementation of diets with fresh apricot, dried apricot and apricot kernel caused marked increase $(\mathrm{p}<0.05)$ in liver and kidney GSH and decrease $(\mathrm{p}<0.05)$ in liver and kidney MDA levels in comparison with APAP-control rats. It is clear from results that, rats fed on fresh and dried apricot (20\%) supplemented diets showed significant elevation $(\mathrm{p}<0.05)$ in liver and kidney GSH, significant reduction $(\mathrm{p}<0.05)$ in liver and kidney MDA levels in a comparison with rats fed on fresh and dried apricot $(10 \%)$ supplemented diets. Moreover, supplementation of diet with apricot kernel $(20 \%)$ caused significant increase $(\mathrm{p}<0.05)$ in liver and kidney GSH levels as compared to apricot kernel $(10 \%)$ supplemented diet, indicating dose dependent effect. While, rats fed on apricot kernel $(10 \%)$ supplemented diet showed marked decrease $(\mathrm{p}<0.05)$ in liver and kidney MDA as compared to rats fed on apricot kernel (20\%) supplemented diet, providing reverse-dose dependent effect.

Table (1): Food intake, body weight gain and relative weight of liver and kidney in experimental groups (mean $\pm \mathrm{SE})$

\begin{tabular}{|r|r|c|c|c|c|}
\hline $\begin{array}{r}\text { Group } \\
\text { No. }\end{array}$ & $\begin{array}{r}\text { Parameters } \\
\text { Groups }\end{array}$ & $\begin{array}{c}\text { Food intake/ rat } \\
(\mathrm{g})\end{array}$ & $\begin{array}{c}\text { Body weight gain } \\
(\mathrm{g})\end{array}$ & $\begin{array}{c}\text { Relative liver } \\
\text { Weight } \\
\%\end{array}$ & $\begin{array}{c}\text { Relative kidney } \\
\text { Weight } \\
\%\end{array}$ \\
\hline G1 & Healthy (-ve control) & $503.75 \pm 13.34^{\mathrm{a}}$ & $130.00 \pm 8.34^{\mathrm{a}}$ & $2.97 \pm 0.17$ & $0.27 \pm 0.013$ \\
\hline G2 & APAP (+ve control) & $507.90 \pm 14.45^{\mathrm{a}}$ & $132.75 \pm 11.79^{\mathrm{a}}$ & $2.72 \pm 0.08$ & $0.26 \pm 0.008$ \\
\hline G3 & Fresh Apricot (10\%) & $544.40 \pm 24.33^{\mathrm{b}}$ & $136.50 \pm 7.61^{\mathrm{b}}$ & $2.82 \pm 0.12$ & $0.24 \pm 0.005$ \\
\hline G4 & Fresh Apricot (20\%) & $606.90 \pm 26.70^{\mathrm{c}}$ & $139.90 \pm 11.87^{\mathrm{b}}$ & $2.78 \pm 0.12$ & $0.24 \pm 0.007$ \\
\hline G5 & Dried Apricot (10\%) & $557.50 \pm 22.52^{\mathrm{d}}$ & $138.75 \pm 7.42^{\mathrm{b}}$ & $2.94 \pm 0.13$ & $0.23 \pm 0.007$ \\
\hline G6 & Dried Apricot (20\%) & $646.25 \pm 28.28^{\mathrm{e}}$ & $145.00 \pm 11.37^{\mathrm{c}}$ & $2.70 \pm 0.15$ & $0.23 \pm 0.011$ \\
\hline G7 & Apricot Kernel (10\%) & $476.90 \pm 24.63^{\mathrm{f}}$ & $116.25 \pm 9.29^{\mathrm{d}}$ & $2.84 \pm 0.14$ & $0.25 \pm 0.007$ \\
\hline G8 & Apricot Kernel (20\%) & $460.62 \pm 24.35^{\mathrm{g}}$ & $98.12 \pm 12.78^{\mathrm{e}}$ & $2.40 \pm 0.13$ & $0.26 \pm 0.014$ \\
\hline
\end{tabular}

$\mathrm{P} \leq 0.05$, there are no significant difference between means have the same letters in the same column 
Table (2): Serum total protein, albumin and bilirubin (total, direct and indirect) levels in experimental groups (mean $\pm \mathrm{SE}$ )

\begin{tabular}{|c|c|c|c|c|c|c|}
\hline \multirow[t]{2}{*}{$\begin{array}{l}\text { Group } \\
\text { No. }\end{array}$} & \multirow{2}{*}{ Parameters Groups } & \multirow[t]{2}{*}{$\begin{array}{l}\text { Total protein } \\
\text { (g/dl) }\end{array}$} & \multirow[t]{2}{*}{$\begin{array}{l}\text { Albumin } \\
\text { (g/dl) }\end{array}$} & \multicolumn{3}{|c|}{$\begin{array}{l}\text { Bilirubin } \\
\text { (mg/dl) }\end{array}$} \\
\hline & & & & Total & Direct & Indirect \\
\hline G1 & Heathy (-ve control) & $8.42 \pm 0.10^{\mathrm{a}}$ & $5.30 \pm 0.06^{\mathrm{a}}$ & $0.48 \pm 0.018^{\mathrm{a}}$ & $0.16 \pm 0.004^{\mathrm{a}}$ & $0.31 \pm 0.015^{\mathrm{a}}$ \\
\hline G2 & APAP (+ve control) & $5.07 \pm 0.28^{b}$ & $3.47 \pm 0.08^{b}$ & $1.43 \pm 0.033^{\mathrm{b}}$ & $0.63 \pm 0.015^{\mathrm{b}}$ & $0.80 \pm 0.028^{\mathrm{b}}$ \\
\hline G3 & Fresh Apricot (10\%) & $6.46 \pm 0.10^{c}$ & $4.32 \pm 0.04^{\mathrm{cf}}$ & $0.85 \pm 0.007^{\mathrm{c}}$ & $0.45 \pm 0.007^{\mathrm{c}}$ & $0.40 \pm 0.008^{\mathrm{cd}}$ \\
\hline G4 & Fresh Apricot (20\%) & $7.19 \pm 0.05^{\mathrm{d}}$ & $4.62 \pm 0.08^{d}$ & $0.77 \pm 0.002^{\mathrm{d}}$ & $0.34 \pm 0.010^{\mathrm{d}}$ & $0.43 \pm 0.011^{\mathrm{d}}$ \\
\hline G5 & Dried Apricot (10\%) & $7.36 \pm 0.04^{\mathrm{de}}$ & $4.63 \pm 0.04^{\mathrm{d}}$ & $0.64 \pm 0.010^{\mathrm{e}}$ & $0.26 \pm 0.006^{\mathrm{e}}$ & $0.38 \pm 0.016^{\mathrm{c}}$ \\
\hline G6 & Dried Apricot (20\%) & $7.77 \pm 0.06^{\mathrm{e}}$ & $4.90 \pm 0.03^{\mathrm{e}}$ & $0.58 \pm 0.005^{\mathrm{f}}$ & $0.21 \pm 0.002^{f}$ & $0.37 \pm 0.005^{\mathrm{c}}$ \\
\hline G7 & Apricot Kernel (10\%) & $6.83 \pm 0.16^{\mathrm{cd}}$ & $4.47 \pm 0.05^{\mathrm{cd}}$ & $0.95 \pm 0.007^{g}$ & $0.59 \pm 0.005^{\mathrm{g}}$ & $0.36 \pm 0.011^{\mathrm{c}}$ \\
\hline G8 & Apricot Kernel (20\%) & $6.49 \pm 0.21^{\mathrm{c}}$ & $4.21 \pm 0.05^{\mathrm{f}}$ & $0.90 \pm 0.015^{\mathrm{h}}$ & $0.55 \pm 0.014^{\mathrm{h}}$ & $0.35 \pm 0.022^{\mathrm{c}}$ \\
\hline
\end{tabular}

$\mathrm{P} \leq 0.05$, there are no significant difference between means have the same letters in the same column

Table (3): Serum AST, ALT, and ALP activities in experimental groups (mean \pm SE)

\begin{tabular}{|c|c|c|c|c|}
\hline $\begin{array}{c}\text { Group } \\
\text { No. }\end{array}$ & $\begin{array}{r}\begin{array}{c}\text { Parameters } \\
\text { Groups }\end{array} \\
\text { G1 }\end{array}$ & $\begin{array}{c}\text { AST } \\
(\mathbf{U} / \mathbf{m l})\end{array}$ & $\begin{array}{c}\text { ALT } \\
(\mathbf{U} / \mathbf{m l})\end{array}$ & $\begin{array}{c}\text { ALP } \\
(\mathbf{I U} / \mathbf{L})\end{array}$ \\
\hline G2 & APAP (+ve control) & $38.11 \pm 0.59^{\mathrm{b}}$ & $22.57 \pm 0.42^{\mathrm{b}}$ & $75.49 \pm 1.01^{\mathrm{b}}$ \\
\hline G3 & Fresh Apricot (10\%) & $28.85 \pm 0.11^{\mathrm{c}}$ & $15.82 \pm 0.08^{\mathrm{c}}$ & $47.95 \pm 0.37^{\mathrm{c}}$ \\
\hline G4 & Fresh Apricot (20\%) & $16.34 \pm 0.24^{\mathrm{d}}$ & $14.51 \pm 0.10^{\mathrm{d}}$ & $45.70 \pm 0.18^{\mathrm{d}}$ \\
\hline G5 & Dried Apricot (10\%) & $28.91 \pm 0.20^{\mathrm{c}}$ & $15.27 \pm 0.10^{\mathrm{c}}$ & $47.21 \pm 0.04^{\mathrm{c}}$ \\
\hline G6 & Dried Apricot (20\%) & $16.02 \pm 0.13^{\mathrm{d}}$ & $13.53 \pm 0.11^{\mathrm{e}}$ & $41.10 \pm 0.23^{\mathrm{e}}$ \\
\hline G7 & Apricot Kernel (10\%) & $16.36 \pm 0.24^{\mathrm{d}}$ & $14.38 \pm 0.15^{\mathrm{d}}$ & $47.65 \pm 0.43^{\mathrm{c}}$ \\
\hline G8 & Apricot Kernel (20\%) & $29.41 \pm 0.10^{\mathrm{c}}$ & $15.60 \pm 014^{\mathrm{c}}$ & $51.38 \pm 0.59^{\mathrm{f}}$ \\
\hline
\end{tabular}

$\mathrm{P} \leq 0.05$, there are no significant difference between means have the same letters in the same column.

Table (4): Serum urea, creatinine, and uric acid levels in experimental groups (mean \pm SE)

\begin{tabular}{|c|c|c|c|c|}
\hline $\begin{array}{c}\text { Group } \\
\text { No. }\end{array}$ & $\begin{array}{r}\text { Parameters } \\
\text { Groups }\end{array}$ & $\begin{array}{c}\text { Urea } \\
(\mathbf{m g} / \mathbf{d l})\end{array}$ & $\begin{array}{c}\text { Creatinine } \\
(\mathbf{m g} / \mathbf{d l})\end{array}$ & $\begin{array}{c}\text { Uric acid } \\
(\mathbf{m g} / \mathbf{d l})\end{array}$ \\
\hline G1 & Heathy (-ve control) & $31.73 \pm 0.37^{\mathrm{a}}$ & $0.53 \pm 0.005^{\mathrm{a}}$ & $3.67 \pm 0.04^{\mathrm{a}}$ \\
\hline G2 & APAP (+ve control) & $66.00 \pm 0.83^{\mathrm{b}}$ & $1.53 \pm 0.007^{\mathrm{b}}$ & $9.21 \pm 0.15^{\mathrm{b}}$ \\
\hline G3 & Fresh Apricot (10\%) & $45.21 \pm 0.22^{\mathrm{c}}$ & $0.89 \pm 0.002^{\mathrm{c}}$ & $6.36 \pm 0.06^{\mathrm{c}}$ \\
\hline G4 & Fresh Apricot (20\%) & $38.04 \pm 0.45^{\mathrm{d}}$ & $0.74 \pm 0.003^{\mathrm{d}}$ & $5.18 \pm 0.02^{\mathrm{d}}$ \\
\hline G5 & Dried Apricot (10\%) & $42.04 \pm 0.28^{\mathrm{e}}$ & $0.68 \pm 0.004^{\mathrm{e}}$ & $5.39 \pm 0.07^{\mathrm{d}}$ \\
\hline G6 & Dried Apricot (20\%) & $34.62 \pm 0.18^{\mathrm{f}}$ & $0.61 \pm 0.005^{\mathrm{f}}$ & $4.77 \pm 0.07^{\mathrm{e}}$ \\
\hline G7 & Apricot Kernel (10\%) & $42.49 \pm 0.45^{\mathrm{e}}$ & $1.05 \pm 0.012^{\mathrm{g}}$ & $6.28 \pm 0.08^{\mathrm{f}}$ \\
\hline G8 & Apricot Kernel (20\%) & $46.60 \pm 0.28^{\mathrm{g}}$ & $1.14 \pm 0.015^{\mathrm{h}}$ & $7.26 \pm 0.09^{\mathrm{g}}$ \\
\hline
\end{tabular}

$\mathrm{P} \leq 0.05$, there are no significant difference between means have the same letters in the same column. 
Table (5): Serum lipids profile (TC, TAG, HDL-C, LDL-C, and VLDL-C) levels in experimental groups $($ mean $+\mathrm{SE})$

\begin{tabular}{|r|r|c|c|c|c|c|}
\hline $\begin{array}{r}\text { Group } \\
\text { No. }\end{array}$ & $\begin{array}{r}\text { Parameters } \\
\text { Groups }\end{array}$ & $\begin{array}{c}\text { TC } \\
(\mathbf{m g} / \mathbf{d l})\end{array}$ & $\begin{array}{c}\text { TAG } \\
(\mathbf{m g} / \mathbf{d l})\end{array}$ & $\begin{array}{c}\text { HDL-C } \\
(\mathbf{m g} / \mathbf{d l})\end{array}$ & $\begin{array}{c}\text { LDL-C } \\
(\mathbf{m g} / \mathbf{d l})\end{array}$ & $\begin{array}{c}\text { VLDL-C } \\
(\mathbf{m g} / \mathbf{d l})\end{array}$ \\
\hline G1 & Heathy (-ve control) & $144.01 \pm 0.65^{\mathrm{a}}$ & $70.76 \pm 0.67^{\mathrm{a}}$ & $63.14 \pm 0.64^{\mathrm{a}}$ & $66.72 \pm 1.10^{\mathrm{a}}$ & $14.65 \pm 0.41^{\mathrm{a}}$ \\
\hline G2 & APAP (+ve control) & $246.37 \pm 0.51^{\mathrm{b}}$ & $136.22 \pm 0.95^{\mathrm{b}}$ & $26.81 \pm 0.69^{\mathrm{b}}$ & $192.31 \pm 0.96^{\mathrm{b}}$ & $27.24 \pm 0.19^{\mathrm{b}}$ \\
\hline G3 & Fresh Apricot (10\%) & $185.80 \pm 0.52^{\mathrm{c}}$ & $100.41 \pm 0.26^{\mathrm{c}}$ & $48.76 \pm 0.23^{\mathrm{c}}$ & $116.96 \pm 0.57^{\mathrm{c}}$ & $20.08 \pm 0.05^{\mathrm{c}}$ \\
\hline G4 & Fresh Apricot (20\%) & $172.61 \pm 0.37^{\mathrm{d}}$ & $91.63 \pm 0.49^{\mathrm{d}}$ & $51.31 \pm 0.35^{\mathrm{d}}$ & $102.98 \pm 0.58^{\mathrm{d}}$ & $18.33 \pm 0.10^{\mathrm{d}}$ \\
\hline G5 & Dried Apricot (10\%) & $165.42 \pm 0.75^{\mathrm{e}}$ & $87.46 \pm 0.44^{\mathrm{e}}$ & $55.47 \pm 0.39^{\mathrm{e}}$ & $92.45 \pm 0.84^{\mathrm{e}}$ & $17.49 \pm 0.09^{\mathrm{e}}$ \\
\hline G6 & Dried Apricot (20\%) & $152.97 \pm 0.37^{\mathrm{f}}$ & $79.61 \pm 0.60^{\mathrm{f}}$ & $59.22 \pm 0.19^{\mathrm{f}}$ & $77.84 \pm 0.45^{\mathrm{f}}$ & $15.91 \pm 0.12^{\mathrm{f}}$ \\
\hline G7 & Apricot Kernel (10\%) & $178.98 \pm 0.19^{\mathrm{g}}$ & $109.90 \pm 0.53^{\mathrm{g}}$ & $46.74 \pm 0.45^{\mathrm{g}}$ & $110.01 \pm 0.61^{\mathrm{g}}$ & $21.98 \pm 0.10^{\mathrm{g}}$ \\
\hline G8 & Apricot Kernel (20\%) & $174.69 \pm 0.40^{\mathrm{h}}$ & $105.26 \pm 0.44^{\mathrm{h}}$ & $42.10 \pm 0.40^{\mathrm{h}}$ & $112.06 \pm 0.78^{\mathrm{g}}$ & $21.05 \pm 0.09^{\mathrm{h}}$ \\
\hline
\end{tabular}

$\mathrm{P} \leq 0.05$, there are no significant difference between means have the same letters in the same column.

Table (6): Liver and kidney GSH and MDA concentrations in experimental groups (mean \pm SE)

\begin{tabular}{|c|c|c|c|c|c|}
\hline $\begin{array}{c}\text { Group } \\
\text { No. }\end{array}$ & Parameters & \multicolumn{2}{|c|}{$\begin{array}{c}\text { Groups } \\
\text { GSH } \\
\end{array}$} & & \multicolumn{2}{|c|}{$\begin{array}{c}\text { MDA } \\
\text { (nmol/g tissue) }\end{array}$} \\
\cline { 3 - 6 } & & Liver & kidney & Liver & kidney \\
\hline G1 & Heathy (-ve control) & $78.63 \pm 0.22^{\mathrm{a}}$ & $72.12 \pm 0.49^{\mathrm{a}}$ & $1.07 \pm 0.02^{\mathrm{a}}$ & $0.45 \pm 0.01^{\mathrm{a}}$ \\
\hline G2 & APAP (+ve control) & $51.23 \pm 0.06^{\mathrm{b}}$ & $49.39 \pm 0.17^{\mathrm{b}}$ & $2.12 \pm 0.04^{\mathrm{b}}$ & $1.11 \pm 0.02^{\mathrm{b}}$ \\
\hline G3 & Fresh Apricot (10\%) & $61.48 \pm 0.42^{\mathrm{c}}$ & $59.03 \pm 0.33^{\mathrm{c}}$ & $1.51 \pm 0.04^{\mathrm{c}}$ & $0.69 \pm 0.01^{\mathrm{c}}$ \\
\hline G4 & Fresh Apricot (20\%) & $63.95 \pm 0.34^{\mathrm{d}}$ & $61.46 \pm 0.07^{\mathrm{d}}$ & $1.56 \pm 0.01^{\mathrm{d}}$ & $0.64 \pm 0.03^{\mathrm{d}}$ \\
\hline G5 & Dried Apricot (10\%) & $66.84 \pm 0.16^{\mathrm{e}}$ & $63.20 \pm 0.03^{\mathrm{e}}$ & $1.38 \pm 0.04^{\mathrm{e}}$ & $0.60 \pm 0.01^{\mathrm{e}}$ \\
\hline G6 & Dried Apricot (20\%) & $72.87 \pm 0.36^{\mathrm{f}}$ & $67.16 \pm 0.44^{\mathrm{f}}$ & $1.22 \pm 0.01^{\mathrm{f}}$ & $0.52 \pm 0.05^{\mathrm{f}}$ \\
\hline G7 & Apricot Kernel (10\%) & $56.22 \pm 0.33^{\mathrm{g}}$ & $55.03 \pm 0.58^{\mathrm{g}}$ & $1.64 \pm 0.05^{\mathrm{g}}$ & $0.73 \pm 0.01^{\mathrm{g}}$ \\
\hline G8 & Apricot Kernel (20\%) & $58.37 \pm 0.02^{\mathrm{h}}$ & $57.95 \pm 0.84^{\mathrm{h}}$ & $1.71 \pm 0.05^{\mathrm{h}}$ & $0.77 \pm 0.01^{\mathrm{h}}$ \\
\hline
\end{tabular}

$\mathrm{P} \leq 0.05$, there are no significant difference between means have the same letters in the same column. 


\subsection{2- Histopathological observations:}

Microscopically, liver section of rats from healthy control group revealed normal histological structure of hepatic lobule. Hepatocytes were arranged in one call thick plates radiating from the central vein towards periphery of the lobule. The irregular spaces between hepatic plates were occupied by liver sinusoids lined by endothelial cells. On the other hand, liver section of rats from APAP- control rats showed congestion of central vein, focal hepatic necrosis, fatty degeneration of hepatocytes, Kupffer cells activation and vaculation of hepatocytes. However, liver sections from rats fed on fresh apricot (10\%) supplemented diet was characterized by cytoplasmic vaculation of some hepatocytes and sinusoidal leucocytosis. While, liver section from rats fed on fresh apricot (20\%) supplemented diet was characterized by vaculation of some periportal hepatocytes. Moreover, liver section from rats fed on dried apricot (10\%) supplemented diet was characterized by some Kupffer cells activation. While, liver from rats fed on dried apricot (20\%) supplemented diet was characterized by some sinusoidal leukocytosis. Furthermore, liver section from rats fed on apricot kernel (10\%) supplemented diet was characterized by some Kupffer cells activation. Also, liver section from rats fed on apricot kernel (20\%) supplemented diet was characterized by hydropic degeneration of some hepatocytes. Figures (1 to 8).

In addition, kidney section from healthy control rats was characterized by normal histological structure of renal parenchyma. On the other hand, kidney section from APAP-control rats showed hypertrophy and vaculation of glomerular tuft and eosinophilic protein cast in the lumen of renal tubules. Moreover, kidney section from rats fed on fresh apricot (10\%) supplemented diet that was characterized by vaculations of some endothelial lining of glomerular tuft. While, kidney section from rats fed on fresh apricot (20\%) supplemented diet showed vaculations of some epithelial lining of renal tubules. Also, kidney section from rats fed on dried apricot (10\%) supplemented diet was characterized by vaculation of some endothelial lining of glomerular tufts. While, kidney section from rats fed on dried apricot (20\%) supplemented diet showed characterized by no histopathological changes. Furthermore, kidney section from rats fed on apricot kernel (10\%) supplemented diet was characterized by vaculation of some endothelial lining of glomerular tufts. Additionally, kidney section from rats fed on apricot kernel (20\%) supplemented diet that was characterized by vaculations of some epithelial lining of renal tubules and endothelial lining of glomerular tuft. Figures (9 to 16). 


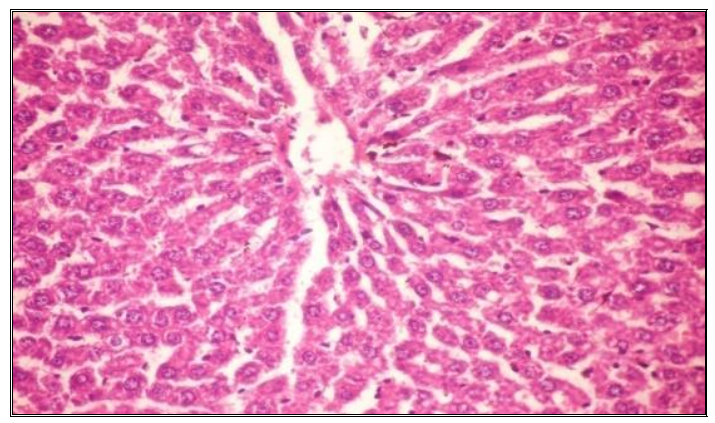

Fig (1): Section of liver from healthy control rats showed normal histological structure of hepatic lobule. ( $\mathrm{H}$ and $\mathrm{E} \times 400$

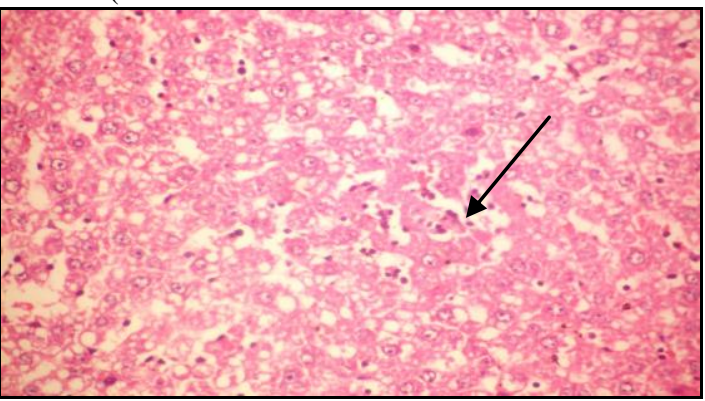

Fig.(3): Section of liver from rats fed on fresh apricot (10\%) supplemented diet that was characterized by cytoplasmic vaculation of some hepatocytes and sinusoidal leucocytosis.

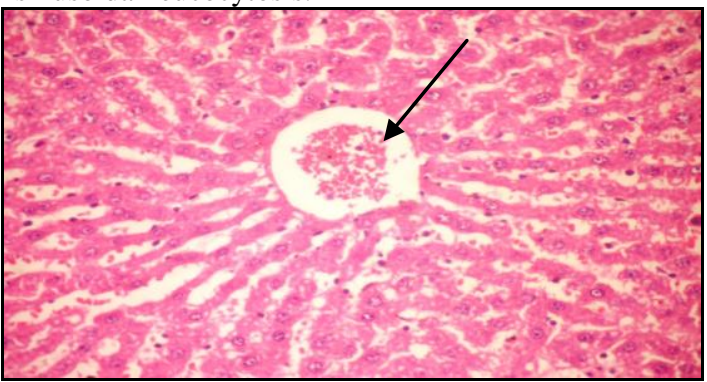

Fig (5): Section of liver from rats fed on dried apricot (10\%) supplemented diet which was characterized by some Kupffer cells activation

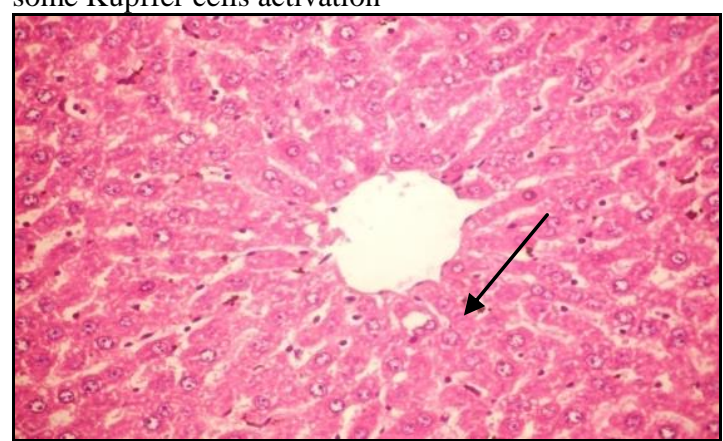

Fig (7): Section of liver from rats fed on apricot kernel (10\%) supplemented diet that was characterized by some Kupffer cells activation.

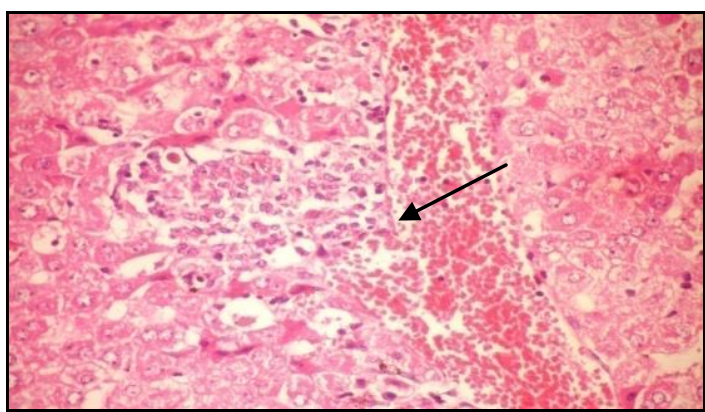

Fig (2): Section of liver from APAP- control rats which characterized by congestion of central vein, focal hepatic necrosis.

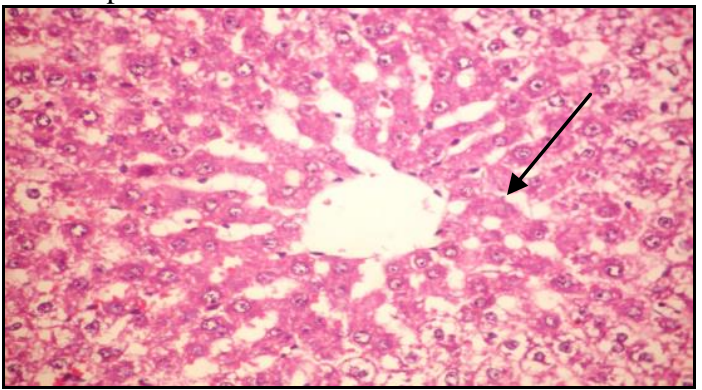

Fig (4): Section of liver from from rats fed on fresh apricot (20\%) supplemented diet that was characterized by vaculation of some periportal hepatocytes

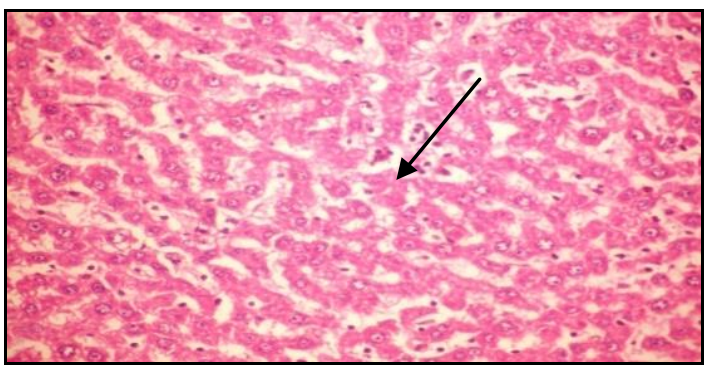

Fig (6): Section of liver from rats fed on dried apricot $(20 \%)$ supplemented diet that was characterized by some sinusoidal leukocytosis.

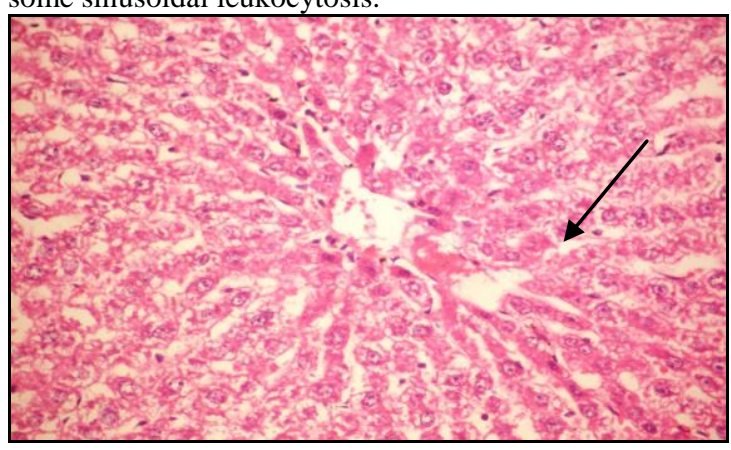

Fig (8): Section of liver from rats fed on apricot kernel (20\%) supplemented diet that was characterized by hydropic degeneration of some hepatocytes. 


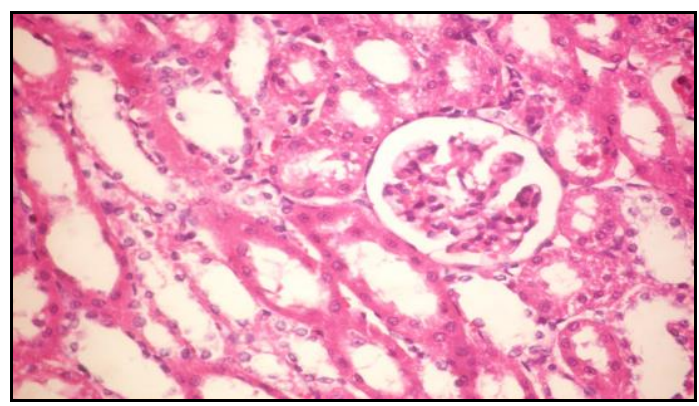

Fig.(9):Section of kidney from healthy control rats which was characterized by normal histological structure of renal parenchyma.

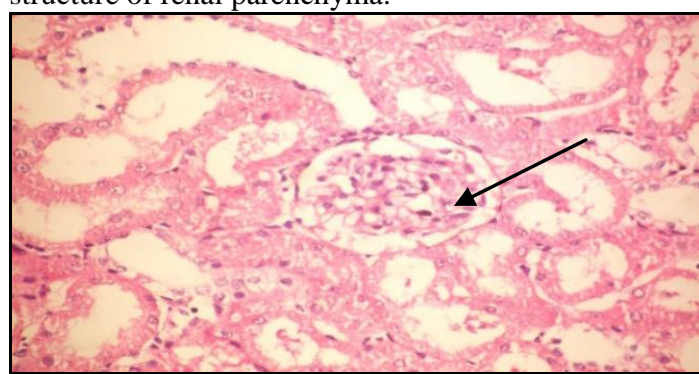

Fig (11): Section of kidney from rats fed on fresh apricot (10\%) supplemented diet that was characterized by vaculations of some endothelial

lining of glomerular tuft.

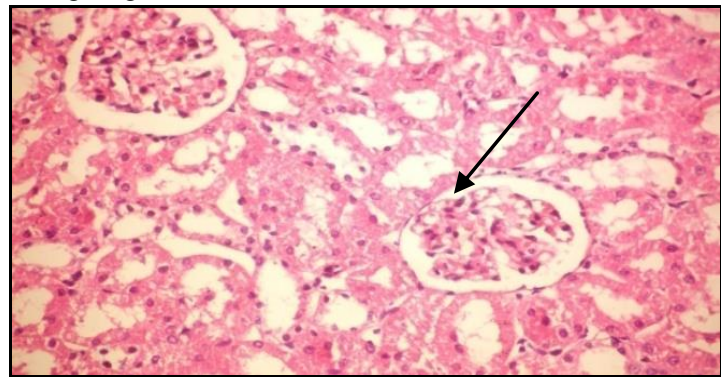

Fig (13): Section of kidney from rats fed on dried apricot (10\%) supplemented diet which was characterized by vaculation of some endothelial lining of glomerular tufts

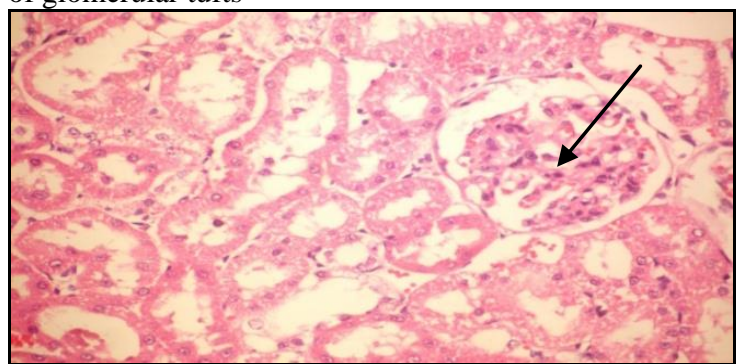

Fig (15): Section of kidney from rats fed on aprico kernel (10\%) supplemented diet that was characterized by vaculation of some endothelial lining of glomerular tufts

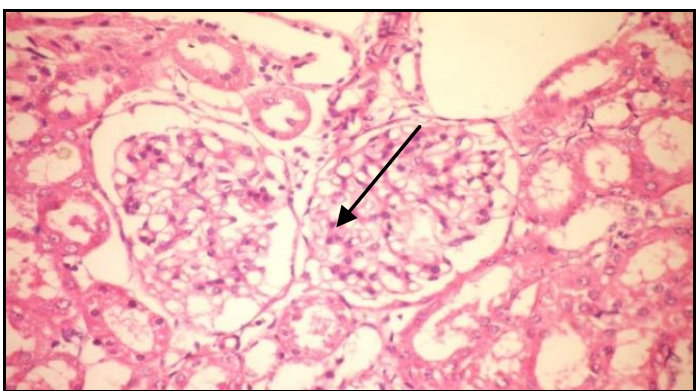

Fig (10): Section of kidney from APAP-control rats

that was characterized by hypertrophy and vaculation of glomerular tuft

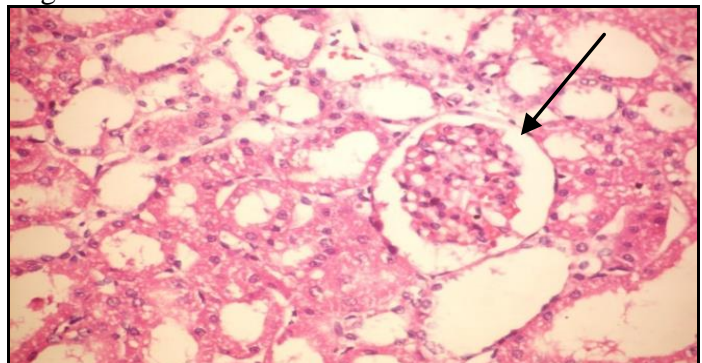

Fig (12): Section of kidney from rats fed on fresh apricot (20\%) supplemented diet that was characterized by vaculations of some epithelial lining of renal tubules.

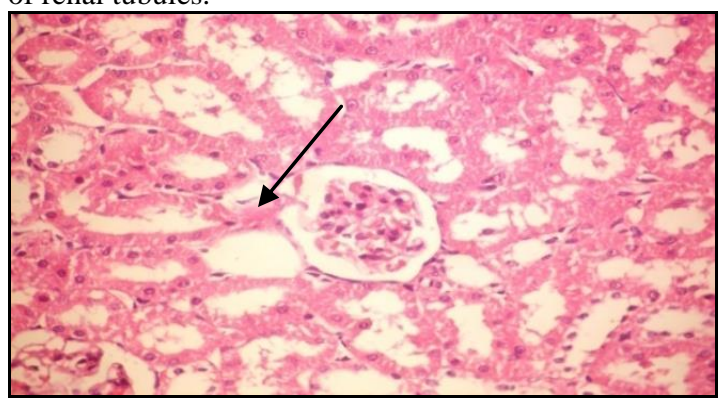

Fig (14): Section of kidney from rats fed on dried apricot (20\%) supplemented diet that was characterized by no histopathological changes.

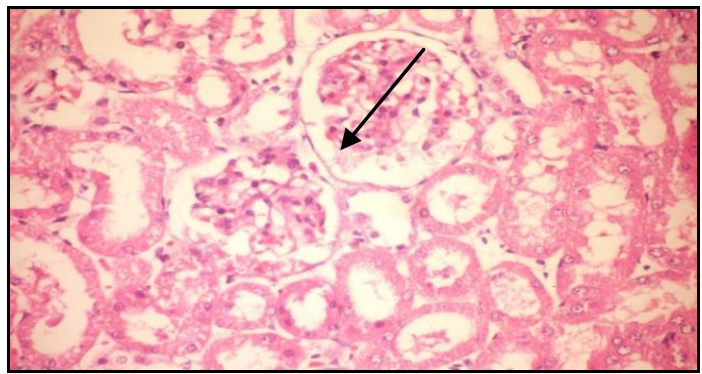

Fig (16): Section of kidney from rats fed on apricot kernel (20\%) supplemented diet that was characterized by vaculations of some epithelial lining of renal tubules and endothelial lining of glomerular tuft. 


\subsection{Discussion:}

In the present study efforts has been made to evaluate possible hepatoprotective and antioxidant activity of apricot and its kernel in APAP-induced hepatic damage in rat model. APAP is considered a predictable hepatotoxin, where biochemical signs of hepatic damage will become apparent within 24 to $48 \mathrm{~h}$ after the time of overdose and produce dose dependent centrilobular necrosis in the liver (Wadekar\& Patil, 2014).

The reduction of serum albumin in paracetamol treated group may be due to formation of protein adduct. Toxic metabolites NAPQI leads to covalent modification of cellular target protein, cell death and organ damage (Parmar et al., 2010). The primary toxicity of APAP is the result of drug metabolism in both the liver and extrahepatic tissues (Gu et al., 2005). Hepatic damage induced by APAP is largely due to biotransformation of a highly reactive and toxic metabolite $\mathrm{N}$-acetyl-P-benzoquinone imine (NAPQI). NAPQI is detoxified by conjugation with reduced glutathione $(\mathrm{GSH})$ to form mercapturic acid. If the rate of NAPQI formation exceeds the detoxification by GSH; it oxidizes tissue macromolecules such as lipid or thiol group in protein and alters the homeostasis of calcium after depleting GSH (Haldar et al., 2012). The NAPQI then causes acylation or oxidation of cytosolic and membrane proteins and generation of reactive oxygen species (ROS). This leads to further oxidation of proteins thiols, lipid peroxidation, DNA fragmentation and ultimately cell necrosis. The important sign of hepatocyte damage is largely to reflect in leaking of cellular enzymes into the bloodstream due to disturbances caused in the transport functions of hepatocytes. Therefore determination of enzymes in the serum is a useful biological marker of the extent and nature of hepatocelluar damage (Sini et al., 2006; and Wadekar \& Patil, 2014). In the present experimental findings, the rats treated with paracetamol $(500 \mathrm{mg} / \mathrm{kg})$, showed a significant hepatic damage, which can be correlated with the increased serum marker enzyme activities (ALT, AST, ALP), bilirubin and MDA formation in liver and kidney homogenates .Serum bilirubin is one of the most sensitive tests employed in the diagnosis of hepatic diseases, hyperbilirubinaemia was observed due to excessive heme destruction and blockage of biliary tract. As a result of hepatic disease there is a mass inhibition of the conjugation reaction and release of unconjugated bilirubin from damaged and dead hepatocytes (Sundari et al., 2011; and Sabiu et al.., 2015).

At therapeutic doses, APAP is metabolized via glucuronidation and sulfation reactions occurring primarily in the liver which result in the water-soluble metabolites that are excreted via the kidney. The result of the metabolic conversion of APAP by the microsomal P-450 enzyme system is that, a highly reactive intermediate, namely, NAPQI is produced. This metabolite is then reduced by GSH. APAP-induced nephrotoxicity may be due to this metabolic activation of APAP to the reactive metabolite, NAPQI. When large doses of APAP are ingested, there is more severe GSH depletion as well as massive production of metabolites, which compounds the toxicity, leaving large amounts of reactive metabolite unbound. These intermediates then form covalent bindings with macromolecules on cellular protein. This process disrupts homeostasis and initiates cell death, leading to tissue necrosis and ultimately to organ dysfunction. The concentration of intracellular GSH, therefore, is the key determinant of the extent of APAP-induced 
renal injury, thus, interest has been focused on compounds that act as antioxidants and are capable of stimulating GSH synthesis (Cekmen et al., 2009).

Pre-treatment with apricot and its kernel at (10 and 20\%) for 42 days followed by paracetamol administration resulted in a significant prevention of elevated serum hepatic marker enzymes and restored total proteins. The prevention of elevated serum marker enzymes by apricot and its kernel may be due to hepatocytes cell membrane stabilization, which then prevents the cystolic release in circulation. These findings are in agreement with the documented fact that, serum transferase activities return to normal with healing of hepatic parenchyma and regeneration of hepatocytes. Furthermore alterations of histoarchitectural changes i.e hepatic lymphocytes infiltration and other inflammatory cells due to paracetamol are prevented with tested fruit in dose dependently. Moreover, apricot significantly reversed the MDA formation to normal range. Hence, it may be possible that the mechanism of hepato-renal protection of apricot is largely due to its antioxidant effect. It is well reported that a deficiency of GSH within organisms can lead to tissue disorder and injury (Wadekar \& Patil, 2014). In the present study, we have demonstrated the effectiveness of the apricot and its kernel in protecting body cells from paracetamol induced cellular damage in rats. Therefore, the levels of glutathione are of critical importance in liver injury caused by paracetamol. The results of the present study was in line with earlier report because we found that pre-treatment with fresh and dried apricots and apricot kernel able to blunted antioxidant system to normal. Biological systems have a number of mechanisms to protect themselves against the damaging effects of reactive oxygen species.

Also, elevated levels of MDA in tissue have been regarded as an indicator for cellular damage due to excess lipid peroxidation processes that occur during malfunction of the antioxidant defense system. Indeed, APAP overdose induces renal oxidative stress as manifested by a decrease in antioxidant enzymes and an increase in lipid peroxidation product (MDA). Lipid peroxidation is an autocatalytic process, which is a common consequences of cell death and its MDA formation is increased due to oxidative stress associated with NAPQI (Elkarib, 2014).

Apricot is a fruit which has a high content of carotenoids, mainly $\beta$-carotene, flavinoids, and phenolic compounds. It also contains vitamins $\mathrm{C}$ and $\mathrm{E}$ and $\mathrm{Se}$ which are well-known dietary antioxidants. $\beta$ - carotene protected human liver cells against oxidantmediated changes in plasma membrane integrity. Carotenoids have singlet oxygen and peroxy radical-quenching properties because of their long chain of conjugated double bonds. Vitamin $\mathrm{C}$ is considered to be one of the most powerful, least toxic natural antioxidants. It is an essential cofactor in the hydroxylation of pro-collagen chains during collagen synthesis. Vitamin $\mathrm{C}$ is an excellent electron donor to free radicals which subsequently quench their deleterious activity on cellular macromolecules, thus playing a role in antioxidant mechanism (Sabiu et al., 2015). Also, vitamin E and Se also have antioxidant properties (Ozturk et al., 2006). Furthermore, $\beta$-carotene treatment along with APAP caused a significant reduction of APAP-induced elevation of AST, ALT, and ALP and appears to be protective in reducing the injurious effect of APAP (Morakinyo et al., 2012).

Administration of APAP showed significant increase in serum creatinine and urea concentrations when compared to control groups, which clearly indicates the intrinsic acute renal failure (Ishola \& Awodele, 2011). Apricot and its kernel prevent the increase 
of APAP induced serum creatinine and urea concentration. Generally, the antioxidant property of apricot and its kernel against pracetamol toxicity may be due to bioactive compounds flavonoids, phenolic compound, pectic polysaccharide. These compounds quench ROS and regenerate membrane-bound antioxidants levels. Also, vitamin C and $\alpha-$ tocopherol supplementation significantly prevents the increase of APAP induced serum creatinine and urea concentrations indicating their effectiveness in ameliorating the APAP induced nephrotoxicity (Kumar et al., 2013).

Paracetamol seems to cause impairment in lipoprotein metabolism and also alteration in cholesterol metabolism. The levels of TC, TAG and LDL-C were significantly increased paracetamol treated rats, when compared to controls. Elevation of TAG during paracetamol intoxication could be due to increased availability of free fatty acids, decreased hepatic release of lipoprotein and increased esterification of free fatty acids (Sundari et al., 2011). Pre-treatment of rats with fresh, dried apricots and apricot kernel significantly decreased serum lipids profile in paracetamol toxicity in rats because of its hypolipidemic effect that may be contributed to their highly content of dietary fibers, carotenes, tocopherols, and polyunsaturated fatty acids.

\section{Conclusion}

The results of present study showed that diet rich in apricot and its kernel protect the cell from APAP-induced toxicity that was manifested by improvement of liver and kidney functions, hyperlipidemia, and oxidative stress. Therefore, it is likely that apricot and its kernel have protective effect on body cells against toxic agents.

\section{References:}

Abirami A., Nagarani G., and Siddhuraju P. (2015): Hepatoprotective effect of leaf extracts from Citrus hystrix and C. maxima against paracetamol induced liver injury in rats, Food Science and Human Wellness; 3:1-17.

Allain C.C., Poon L.S., Chan C.S.G., Richmond W. and Fu P.C. (1974): Enzymatic determination of total serum cholesterol. Clinical Chemistry; 20 (4): 470-475.

Barham D. and Trinder P. (1972): An improved colour reagent for the determination of blood glucose by the oxidase system. Analyst; 97: 142-145.

Bartels H., Bohmer M., and Hierli C. (1972): Serum creatinine determination without protein precipitation. Clin. Chim. Acta; 37: 193-197.

Belfield A., and Goldberg D.M. (1971): Colourimetric determination of alkaline phosphatase activity. Enzyme; 12: 561-568.

Beutler E., Dutron O., and Kelly B.M. (1963): Improved method for the determination of blood glutathione. J. Lab and Clin. Med.; 61(5): 882-888. 
Bhadauria M. (2010): Dose-dependent heptoprotective effect of emodin against acetaminophen induced acute damage in rats. Experimental and Toxicologic pathology; 62: 627-635.

Cekmen M, Ilbey Y.O. , Ozbek E., Simsek A., Somay A., and Ersoz C. (2009) : Curcumin prevents oxidative renal damage induced by acetaminophen in rats Food and Chemical Toxicology; 47 : 1480-1484.

Das J., Ghosh J., Manna P., and Sil P.C. (2010): Taurine protects acetaminopheninduced oxidative damage in mice kidney through APAP urinary excretion and CYP2E1 inactivation. Toxicology 269: 24-34.

Doumas B.T., Watson W.A., and Biggs H.G. (1971): Albumin standards and measurement of serum albumin with bromocreal green. Clin. Chim Acta; 31: 87-96.

Draper H.H., and Hadley M. (1990): MDA determination as an index of lipid peroxidation. Methods Enzymol; 186: 421-430.

Drury R.A., and Wallington E.A. (1980): Carleton's histology technique. $4^{\text {th }}$ Edn.Oxford university press, Newyork.

Durmaz G., and Alpaslan M. (2007): Antioxidant properties of roasted apricot (Prunus armeniaca L.) kernel. Food Chemistry; 100: 1177-1181.

Elkarib A. O. (2014): Impact of Dehydro-epiandrosterone in Prevention of Paracetamol induced Nephrotoxicity in Rats. American Medical Journal; 5 (1): 16-27.

Fawcett J.K. and Scott J.E. (1960): A rapid and precise of method for the determination of urea. J, Clin., Path; 13: 156-160.

Fossati P., and Principe L. (1982): Serum triglycerides determined colorimetrically with an enzyme that produces hydrogen peroxide. Clin. Chem; 28(10): 2077-2080.

Fridewald W.T. (1972): Determination of lioproteins in serum. Clin. Chem; 18: 499.

Gornall A.C., Bardawill C.J., and David M.M. (1949): Determination of serum proteins by means of the biuret reaction. J. Biol. Chem; 177: 751-766.

Gu J., Cui H., Behr M., Zhang Q.Y., Yang W., Hinson J.A., and Ding X. (2005): In vivo mechanisms of tissue-selective drug toxicity: effects of liver-specific knockout of the NADPH-cytochrome P-450 reductase gene on acetaminophen toxicity in kidney, lung, and nasal mucosa. Mol. Pharmacol.; 67: 623-630. 
Gul H., Uysal B., Cakir E., Yaman, H., Macita, E., Yildirim, A.O., Eyi, Y. E., Kaldirim, U., Oztas, E., Akgul, E. O., Cayci, T., Ozler, M., Topal, T., Oter, S., Korkmaz, A., Toygar, M., and Demirba, S. (2012): The protective effects of ozone therapy in a rat model of acetaminophen- induced liver injury. Enviromental Tox. \& Pharmacology; 34: 81-86.

Haldar P.K., Biswas M. Bhhhattacharya S., Karan T.K. and Ghosh A.K. (2012): Hepatoprotective activity of Dregea Volubilis fruit against paracetamol- induced liver damage in rats. Indian journal of pharmaceutical Education and Research; 46:17-21.

Ishola I.O and Awodele O. (2011): Protective role of Ascorbic acid and AlphaTocopherol against Acetaminophen- induced Nephrotoxicity in rats. AJPSP; 1(1): 75-90.

Kantham S. (2011): Influence of carica Papaya linn extracts on paracetamol and thioacetamide induced hepatic damage in rats. The Internet Journal of Pharmacology; 9(1): 1531-2976.

Kumar E. K., Harsha K.N, Neelakanta R. N, Sudheer V., and Giri Babu N. (2013): In vitro antioxidant activity and in vivo hepatoprotective activity of aqueous extract of Allium cepa bulb in paracetamol induced liver damage in Wistar rats. Research Journal of Pharmaceutical, Biological and Chemical Sciences; 4 (2): 335-343.

Liu Y.T., Lu B.N., and Peng J.Y. (2011): Hepatoprotective activity of the total flavonoids from Rosa laevigata Michx fruit in mice treated by paracetamol. Food chemistry; 125: 719-725.

Morakinyo A.O., Iranloye B.O., Oyelowo O.T., Nnaji J. (2012): Anti-oxidative and hepatoprotective effect of beta-carotene on acetaminophen-induced liver damage in rats. Biology and Medicine; 4 (3): 134-140.

Orhan-Erdogan L., and Kartal M. (2011): Insights into research on phytochemistry and biological activities of Prunus armeniaca L. (apricot). Food Res.Int.;44: 1238-1243.

Ozturk F., Ozturk I.C., Batcioglu K., and Vardi N.(2006): The effect of melatonin on 7,12-dimethyl-benz[a ]anthracene injury in comparisons with vitamin $\mathrm{E}$ and selenium in mouse kidneys. Fundam Clin Pharmacol; 20: 359-364.

Parmar S. R., Vashrambhai P.H., and Kalia k. (2010): Hepatoprotective Activity of Some Plants Extract against Paracetamol Induced Hepatotoxicity in Rats. Journal of Herbal Medicine and Toxicology; 4: (2) 101-106.

Reeves G., Nielsen F.H., and Fahey J.G.C. (1993): AIN-1993 reformulation of the AIN-76: Purified final report of the American Institute of Nutrition Purified Ad Hoc Writing Committee on the reformulation of AIN-76A rodent diet. American Institute, J. Nutr; 26: 1939-1951. 
Reitman S., and Frankel S. (1957): Colourimetric determination of serum GOT [glutomic oxaloacetic transaminase] activity. American Journal Clinical pathology; 28: 56.

Sabiu S., Sunmonu T. O., Ajani E. O., and Ajiboye T. O. (2015): Combined administration of silymarin and vitamin $\mathrm{C}$ stalls acetaminophen-mediated hepatic oxidative insults in Wistar rats. BJP; 82: 1-6.

Sini S., Latha P.G., Sasikumar J.M., Rajashekaran S., Shyamal S. and Shine V.J.(2006): Hepatoprotective studies on Hedyotis Corymbosa. Journal of Ethanopharmacology; 106: 245-249.

Steel R.G.D., and Torrie J.H. (1980): Analysis of covariance, in: principles and procedures of statistics: a biometrical approach; 401-737.

Sundari K., Govindaraju G. and Bharathi B. (2011): Hepatoprotective effect of ethanolic extract of Sphaeranthus indiicus (Linn) on paracetamol- induced liver toxicity in rats. IJABPT; 2:315-321.

Wadekar R. R. and Patil K. S. (2014):Hepatoprotective activity of Uvaria Narum in paracetamol-induced hepatic damage in rats: a biochemical and histopathological evaluation. International Journal of Pharmcognosy; 1(2): 119-129.

Walter $M$ and Gerade RW (1970): Bilirubin direct /total determination. Microchem.J.; 15:231-233.

Yurt B., and Celik I. (2011): Hepatoprotective effect and antioxidant role of sun, sulphited-dried apricot (Prunus armeniaca L.) and its kernel against ethanol-induced oxidative stress in rats. Food and Chemical Toxicology; 49: 508-513.

Zhang J., Gu H.D., Zhang L., Tian Z.J., Zhang Z.Q., Shi X.C., and Ma W.H. (2011): Protective effects of apricot kernel oil on myocardium against ischemia-reperfusion injury in rats. Food and Chemical Toxicology; 49: 3136-3141. 


\section{الملخص باللغة العربية}

التأثير الوقائي للمشمش(Prunus armeniaca L)

ونواته ضد تلف خلايا الكبد والكلى المحاث بالئل بواسطة البار اسيتامول في الجرذان

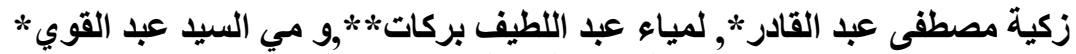

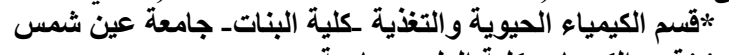

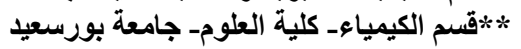

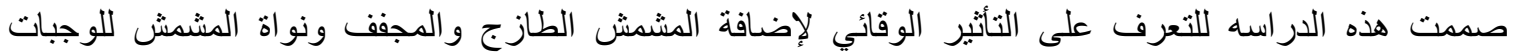

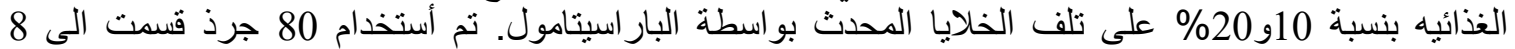

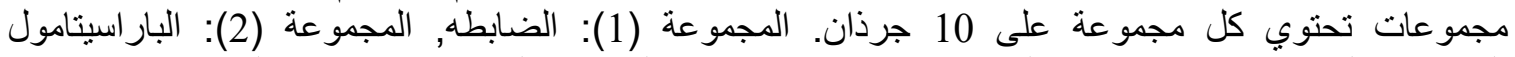

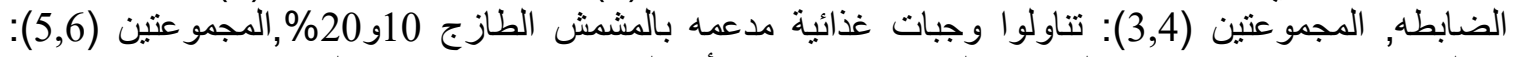

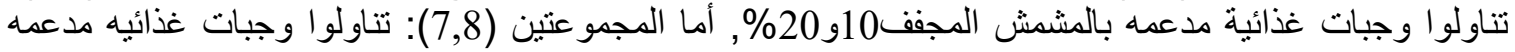

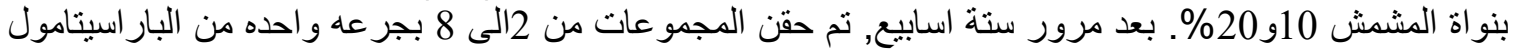

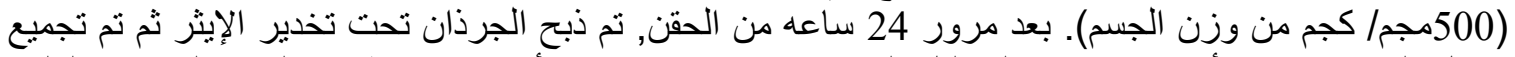

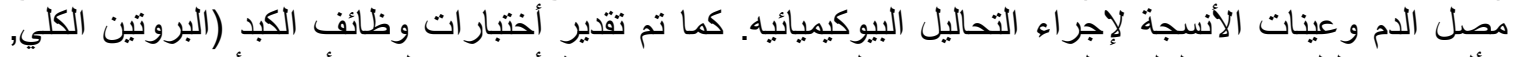

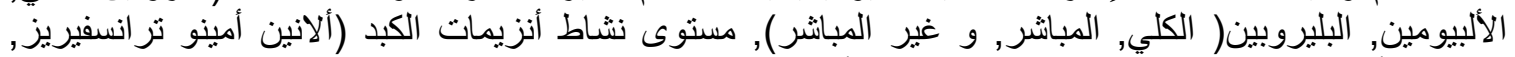

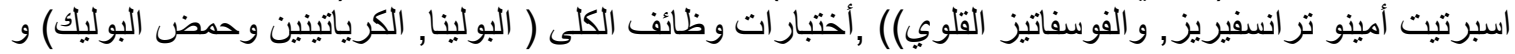

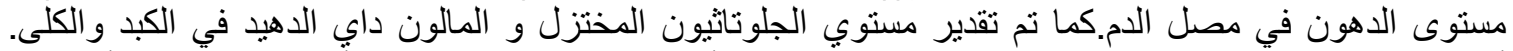

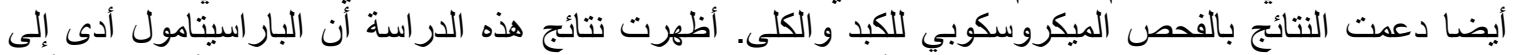

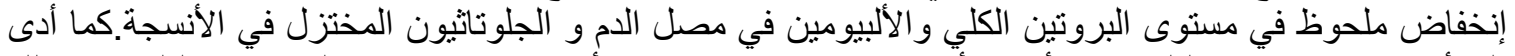

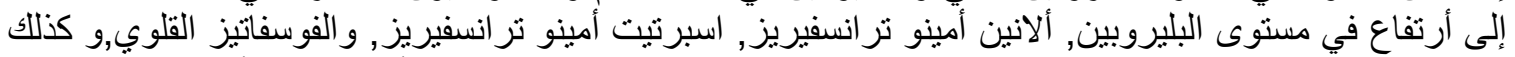

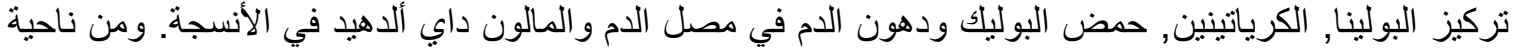

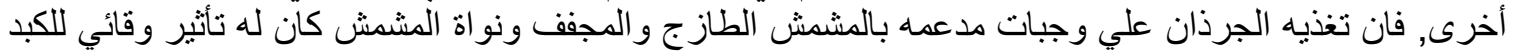

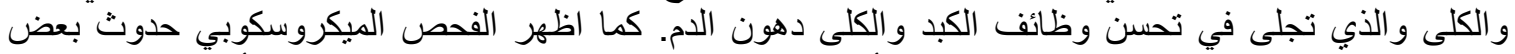

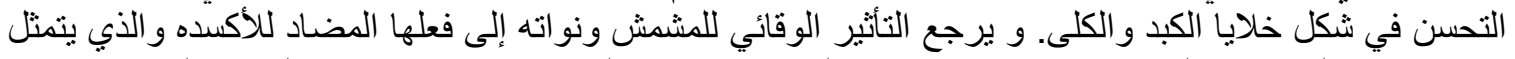

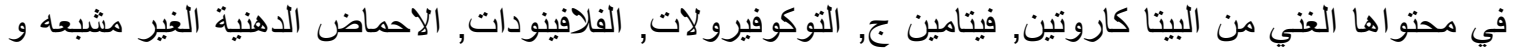
بعض الفينو لات العديده والتي تقي من الثوارد الحره وسمية البار اسيتامول. 